\title{
THEORETICAL PHYSICS
}

\section{Walk the Planck}

\section{Where relativity and quantum mechanics clash, new laws of physics should emerge.}

\section{Giovanni Amelino-Camelia}

The Planck scale is where general relativity and quantum mechanics should clash. It is the realm of the unfeasibly energetic and the unimaginably tiny. It is where the laws of nature are expected to achieve their highest level of elegance and simplicity - and where speculation abounds.

Albert Einstein's general theory of relativity follows a completely different logic from quantum mechanics. In relativity, observables evolve smoothly and deterministically. Quantum mechanics, in contrast, relies on quanta and probabilistic predictions.

Mostly, these differences are moot. Quantum mechanics describes the interactions of microscopic entities, such as the low-energy particles typically studied in laboratories. Here, general relativity can be ignored because the electroweak and electrostrong forces far outweigh gravitational forces. General relativity takes centre stage for the motions of macroscopic bodies such as planets. Here gravitational interactions dominate, because the bodies are composed of a large number of particles; the electroweak and electrostrong forces can be disregarded because they tend to average out.

Around the Planck scale - equivalent to $10^{19}$ gigaelectronvolts - things get tricky. For microscopic particles with energy this high or higher, both quantummechanical and general-relativistic effects come into play. Gravity enters the picture, in other words.

Right now we can only speculate about the outcome of such a clash - current particle accelerators produce energies $10^{16}$ times too low. Some believe that general relativity would adapt. At or near the Planck scale, they argue, gravitational interaction would become quantized. All interactions would be subject to a unified description governed by quantum mechanics. In this scenario, the Planck scale marks the beginning of a new regime for the laws of physics. It collapses our current theories' disorderly multiplicity of interactions into one law of interaction.

String theory provides a fascinating alternative scenario. It can accommodate a form of duality between the properties of a particle with energy a given amount

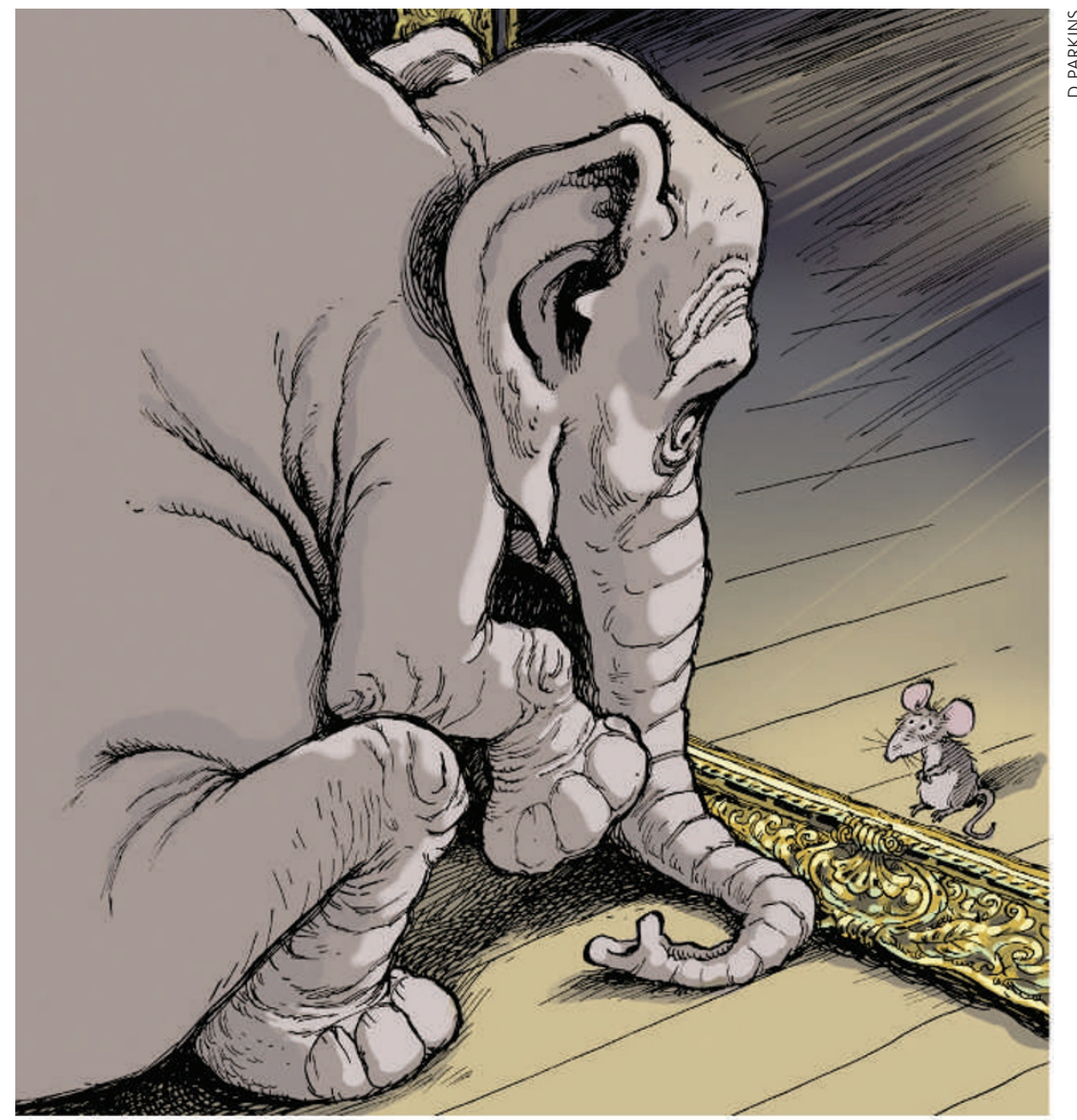

In string theory, the laws of physics above the Planck scale are a mirror image of those below it.

below the Planck scale and the properties of a particle with energy a corresponding amount above the Planck scale. This may happen when the theory is formulated with extra space dimensions, undetected by our senses because of their tiny size. In this line of thinking, the Planck scale still marks the beginning of a new physics, but string theory's 'beyondplanckian' regime is just a mirror copy of the old 'sub-planckian' one.

There is a third, even more baffling, scenario. This posits that the Planck scale is the maximum possible energy of a fundamental particle, just as the speed of light is the maximum possible velocity. As a result, some new effects, such as certain particlephysics reactions forbidden in our current (pre-planckian) theories, would become more significant as the energies of the fundamental particles involved approach the Planck scale.
Preliminary studies suggest that theories based on this Planck-scale energy limit might provide a 'fair' outcome for the clash. General relativity and quantum mechanics would at last be unified in such a way as to preserve some key features of both: the core concepts of relativity would be responsible for the emergence of the Planck-scale energy limit; quantum mechanics would have an important role in describing space-time structure.

Over the past few years, there has been an increased effort to devise ways to seek indirect experimental evidence to discriminate between these alternative scenarios. Some promising ideas are being developed, but it is a truly formidable challenge. The laws of physics at the Planck scale may remain nature's well-kept secret for a very long time.

Giovanni Amelino-Camelia is in the Department of Physics, University La Sapienza, and the Sezione Roma1 of the National Institute for Nuclear Physics, Piazzale Moro 2, Rome 00185, Italy. 